Sabine L. Vrancken*, Arno F. van Heijst, Jeroen C. Hopman, Kian D. Liem, Johannes G. van der Hoeven and Willem P. de Boode

\title{
Detection and quantification of left-to-right shunting using transpulmonary ultrasound dilution (TPUD): a validation study in neonatal lambs
}

DOI 10.1515/jpm-2015-0310

Received September 6, 2015. Accepted March 31, 2016. Previously published online May 9, 2016.

\section{Abstract}

Objectives: We investigated the accuracy of left-to-right shunt detection using transpulmonary ultrasound dilution (TPUD) and compared the agreement between pulmonary over systemic blood flow $(\mathrm{Qp} / \mathrm{Qs})$ ratio measured by TPUD [Qp/Qs(tpud)] and ultrasonic flow probes [Qp/Qs(ufp)].

Methods: Seven newborn lambs under general anesthesia were connected to the TPUD monitor (COstatus ${ }^{\mathrm{TM}}$ ) after insertion of arterial and central venous catheters. A Gore-Tex ${ }^{\circledR}$ shunt, inserted between the descending aorta and left pulmonary artery, was intermittently opened and closed while cardiac output was varied by blood withdrawals. Flow probes were placed around the main pulmonary artery (Qufp) and the descending aorta proximal (Qpre) and distal (Qpost) to the shunt insertion. Qp/ Qs(ufp) was calculated as (Qufp+Qpre-Qpost)/Qufp.

Results: Seventy-two paired measurement sessions were analyzed. Shunts were detected by TPUD with a positive predictive value of $86 \%$, a negative predictive value of $100 \%$, a sensitivity of $100 \%$ and a specificity of $83 \%$. The Bland-Altman analysis comparing Qp/Qs(tpud) and

\footnotetext{
*Corresponding author: Sabine L. Vrancken, Department of Paediatrics - Neonatology, Radboud University Medical Centre, Internal postal code 804, P.O. Box 9101, 6500 HB Nijmegen, The Netherlands, Tel.: +31-24-3614430, Fax: +31-24-3616428, E-mail: s.vrancken@radboudumc.nl

Arno F. van Heijst, Kian D. Liem and Willem P. de Boode: Department of Paediatrics - Neonatology, Radboud University Medical Centre, Nijmegen, The Netherlands

Jeroen C. Hopman: Department of Radiology, Medical Ultrasound Imaging Centre, Radboud University Medical Centre, Nijmegen, The Netherlands

Johannes G. van der Hoeven: Department of Pediatric Intensive care, Radboud University Medical Centre, Nijmegen, The Netherlands
}

Qp/Qs(ufp) showed an overall mean bias (SD) of 0.1 (0.3), limits of agreement (LOA) of \pm 0.6 and a percentage error of $34.8 \%$.

Conclusions: The qualitative diagnostic accuracy of TPUD for shunt detection is high. Modification of the algorithm seems required as shunt quantification by TPUD is accurate, but not yet very precise.

Keywords: Cardiac output; ductus arteriosus; infant; leftto-right shunt; newborn; patent; transpulmonary ultrasound dilution.

\section{Introduction}

Patients in neonatal intensive care units often present with intra- or extracardiac shunts due to cardiac anomalies or failure of adequate postnatal transition [persisting pulmonary hypertension, patent ductus arteriosus (PDA)]. Especially in premature infants, the incidence of PDA with left-to-right shunt is high [1, 2]. A hemodynamic significant patent ductus arteriosus (hsPDA) is associated with increased morbidity (pulmonary edema, increased risk of pulmonary hemorrhage, bronchopulmonary dysplasia, intraventricular hemorrhage, necrotizing enterocolitis and renal failure) due to an increase in pulmonary blood flow and a decrease in systemic blood flow caused by a steal of blood from the systemic into the pulmonary circulation [3-6]. Early and accurate diagnosis of hsPDA and subsequent early treatment might reduce these complications. However, nowadays, no uniform criteria are available for diagnosing hsPDA. Therefore, a combination of repetitive clinical evaluation, echocardiographic studies and biochemical markers [B-type natriuretic peptide (BNP) and its N-terminal fragment, NT-proBNP] is used to individualize management $[5,7,8]$. Echocardiography is the clinical gold standard for the assessment of an hsPDA using measurements of left heart dimension, shunt 
directions and ductal diameter. A review of the literature shows a wide variety in the echocardiographic definition of hsPDA [9-12], and the optimal timing for screening remains unclear. Although there is a widespread interest in functional echocardiography among neonatologists, the method is not yet used as a standard diagnostic tool, is not always bedside available and has a considerable operator variability [13].

Assessing the magnitude of the left-to-right shunt fraction (pulmonary over systemic blood flow ratio $=Q p /$ Qs) might be preferable in order to determine if a PDA is hemodynamically significant as low systemic blood flow is associated with impaired end-organ perfusion [14]. Advanced hemodynamic monitoring using transpulmonary ultrasound dilution (TPUD) technology not only measures the actual cardiac output (systemic blood flow) and hemodynamic volumes, but also detects and quantifies (ductal) shunts [expressed as (Qp/Qs)]. Detection and quantification of a left-to-right shunt could be a helpful tool in the diagnosis and treatment of hsPDA. TPUD is based on changes in ultrasound velocity in arterial blood after central venous injection of isotonic saline at body temperature [15]. As the technique uses an extracorporeal loop that is connected in between an indwelling central venous and arterial catheter of any type, TPUD is applicable in (preterm) neonates without technical restraints. TPUD technology has been validated and found reliable for estimation of cardiac output in adults $[16,17]$, children [18-20] and newborn lambs with and without a leftto-right shunt and acute lung injury [21-23]. Recently, authors demonstrated TPUD to be an accurate tool to detect (small) intracardiac shunts when compared with echocardiography perioperatively in children $[24,25]$.

The objective of this study was to analyze the sensitivity and specificity of shunt detection by TPUD and to compare the agreement of Qp/Qs measurement by TPUD $[Q p / Q s(t p u d)]$ and Qp/Qs measurement by ultrasonic transit time flow probes [Qp/Qs(ufp)] - which is considered the gold standard - in a neonatal lamb model with an artificial left-to-right shunt under different levels of cardiac output.

\section{Methods}

\section{General}

The initial study protocol was designed to validate only cardiac output measurements using TPUD in an animal model with a left-toright shunt under different hemodynamic conditions [22]. The data obtained for this study were collected during the initial study. The study was conducted in accordance with the Dutch national legislation and the principles of the Declaration of Helsinki concerning guidelines for the care and use of laboratory animals, approved by the Ethical Committee on Animal Research of the Radboud University Nijmegen (RU-DEC=2008-117) and performed in seven lambs [3.5-8.3 kg (median $6.5 \mathrm{~kg}$ ), 5-21 days (median 12 days)] under general anesthesia. Premedication consisted of the intramuscular (i.m.) administration of ketamine $(10 \mathrm{mg} / \mathrm{kg})$, atropine $(0.03 \mathrm{mg} / \mathrm{kg})$, midazolam $(0.2 \mathrm{mg} / \mathrm{kg})$ and intravenous (i.v.) administration of propofol $(2 \mathrm{mg} / \mathrm{kg})$. The lambs were orotracheally intubated [cuffed endotracheal tube (ID 4-5 mm; Kruse, Marslev, Denmark)], and the lungs mechanically ventilated in a pressure-controlled mode aiming for tidal volumes of $10 \mathrm{~mL} / \mathrm{kg}$ using a Datex Ohmeda Excel 210 SE anesthesia machine (GE Healthcare, Waukesha, WI, USA). Anesthesia was maintained with isoflurane inhalation (0.5-2.0 vol\%) and continuous i.v. administration of sufentanyl $(15-20 \mu \mathrm{g} / \mathrm{kg} / \mathrm{h})$, midazolam $(0.2 \mathrm{mg} / \mathrm{kg} / \mathrm{h})$ and pancuronium $(0.02 \mathrm{mg} / \mathrm{kg} / \mathrm{h})$ after a loading dose of $0.05 \mathrm{mg} / \mathrm{kg}$. The ventilator settings were adjusted during and after thoracotomy in order to maintain normoxemia $\left(\mathrm{SaO}_{2} 90 \%-95 \%\right)$ and normocapnia $\left(\mathrm{PaCO}_{2} 4.0-6.0 \mathrm{kPa}\right.$; end-tidal $\mathrm{CO}_{2} 4.0-6.0 \mathrm{kPa}$ ). The depth of anesthesia was repeatedly assessed by clinical parameters (heart rate, spontaneous ventilation or elevated arterial pressure) and pain stimuli and was adjusted when necessary. At the end of the experiment, the animals were euthanized using a lethal dose of pentobarbital $(150 \mathrm{mg} / \mathrm{kg})$ intravenously.

\section{Instrumentation}

Intravascular catheters were surgically inserted via the femoral vessels. A double-lumen central venous catheter $(16 \mathrm{G} / 16 \mathrm{~cm} / 1.7 \mathrm{~cm}$, Arrow, Arrow International, Reading, PA, USA) was positioned with the tip in the inferior vena cava. One lumen was used for connection with the venous limb of the AV-loop for TPUD measurement. The other lumen was used for administration of fluids and medication. The tip of the arterial catheter $(16 \mathrm{G} / 13 \mathrm{~cm} / 1.7 \mathrm{~mm}, 681002$, Secalon Tтм, Becton, Dickinson and Company, Oxford, UK) was positioned in the abdominal aorta and connected with the arterial limb of the extracorporeal circuit for TPUD measurement. Another arterial catheter was inserted in the opposite femoral artery to measure blood pressure continuously. A non-stretch, thin-walled vascular graft (ID 4-6 mm, Gore-Tex ${ }^{\circledR}$, W.L. Gore \& Associates Inc., AZ, USA) was then inserted between the descending aorta and the left pulmonary artery through a left-sided thoracotomy. The native ductus arteriosus was closed in all animals. After a loading dose of heparin (100-120 IU/ $\mathrm{kg}$ ), continuous infusion of heparin $(50-100 \mathrm{IU} \mathrm{kg} / \mathrm{h}$ ) was used to prevent shunt thrombosis. In order to measure $Q p / Q$ s ratio and shunt flow, perivascular ultrasonic flow probes (PAX series, Transonic Systems Inc., Ithaca, NY, USA) were placed around the main pulmonary artery (Qufp) and proximal (Qpre) and distal (Qpost) to the aorto-pulmonary shunt on the descending aorta.

\section{Protocol}

The study protocol was started after a stabilization period of $15 \mathrm{~min}$ (Figure 1). During the experiment, the aorto-pulmonary shunt was intermittently closed and opened by (un)clamping. Changes in cardiac output were created using hemorrhagic hypotension by three 


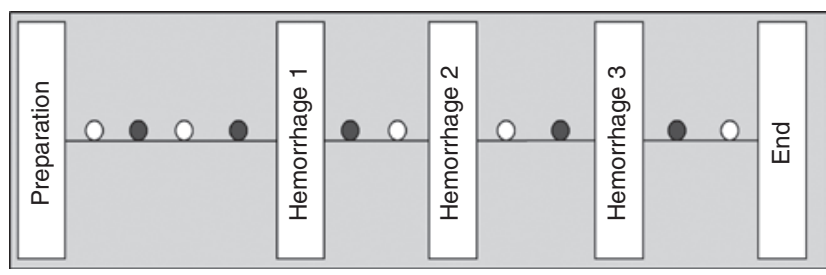

Figure 1: Study protocol.

Circles indicate the timing of three consecutive paired hemodynamic measurements (white circles=measurements with shunt open, gray circles $=$ measurements with shunt closed).

successive periods of blood withdrawals (mean $6 \pm 2 \mathrm{~mL} / \mathrm{kg} /$ withdrawal; mean $19 \pm 3 \mathrm{~mL} / \mathrm{kg}$ in total), aiming for a decrease in mean arterial blood pressure by $10 \mathrm{~mm} \mathrm{Hg} /$ withdrawal over a period of approximately 5 min. Following each intervention, arterial blood gases were obtained and ventilator settings adjusted if necessary. After a stabilization period of $15 \mathrm{~min}$, one session of TPUD measurements - consisting of three consecutive injections with $1.0 \mathrm{~mL} / \mathrm{kg}$ isotonic saline - was performed. Operators performing TPUD measurements were not blinded for the shunt status. As the COstatus (Transonic Systems Inc., Ithaca, NY, USA) automatically calculates Qp/Qs, it was not possible to influence TPUD results.

\section{Transpulmonary ultrasound dilution (TPUD)}

TPUD technology (Figure 2) is operator independent and uses an extracorporeal arteriovenous loop (priming volume $2.7 \mathrm{~mL}$ for neonates) that can be connected to any indwelling arterial and central venous catheter, including umbilical catheters. A peristaltic pump prevents stasis of blood and provides stable blood flow $(6-12 \mathrm{~mL} /$ $\min$ ) during measurement sessions. The technique is based on differences in ultrasound velocity between blood (1570-1585 m/s) and isotonic saline (used as indicator) $(1533 \mathrm{~m} / \mathrm{s})$. Isotonic saline at body temperature is injected into the venous limb of the AV-loop and

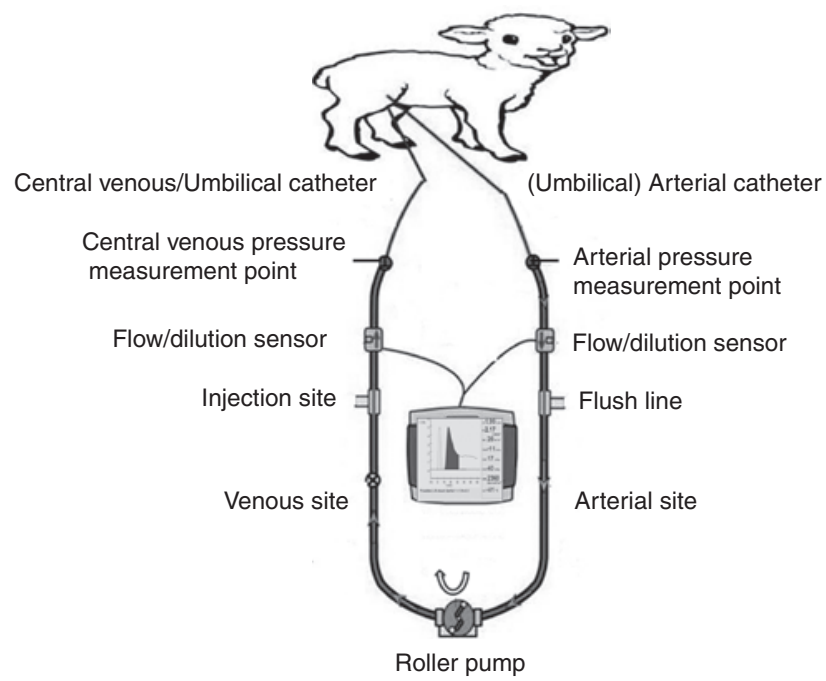

Figure 2: Illustration of the transpulmonary ultrasound dilution method. detected by the venous sensor situated nearby the injection site. A venous dilution curve (Figure 3, light gray curve) is produced as a result of a decrease in blood ultrasound velocity. The COstatus software calculates the exact injected volume. The saline passes the venous catheter, mixes with blood while entering the inferior vena cava and the right heart, passes through the lungs and the left heart and finally arrives in the abdominal aorta where the arterial catheter is situated. The arterial sensor - positioned at the arterial side of the AV-loop - measures the decrease in ultrasound velocity in the blood giving the concentration of the injected indicator and a dilution curve is obtained (Figure 3, dark gray curve). After three measurements (one session), the loop is flushed and the pump stopped.

Cardiac output, hemodynamic volume values and - in case of a detected shunt - Qp/Qs ratio are displayed on the COstatus ${ }^{\mathrm{TM}}$. The calculation of the cardiac output and volumes is based on the obtained ultrasound dilution curve(s) and generated by the COstatus software, first described by Krivitski et al. [15].

In the presence of a left-to-right shunt, part of the indicator makes an extra passage through the lungs before reaching the arterial catheter. This prolongs the disappearance time of the dilution curve. Therefore, the dilution curve has a normal appearance time, a decreased amplitude and an asymmetry in the downslope versus upslope in comparison with a dilution curve without shunts (Figure 3B). Detection and quantitative assessment of $\mathrm{Qp} / \mathrm{Qs}$ is based on a mathematical model of proportionality of increased asymmetry, using the appearance time (Ta) [=time from the beginning of the venous injection to the beginning of the dilution curve(s)] and asymmetry of the dilution curve [=ratio of total full width at half maximum height (b) and the upslope of the curve at half maximum height (\%) (a)] (Figure 3B) [26]. As the calculated area under the obtained curve is larger than the area of the predicted normal dilution curve in the presence of a left-to-right shunt, the Qp/Qs ratio will be $>1$. TPUD was considered to have identified a shunt only if two or more measurements within one session suggested the presence of the shunt.

\section{Other measurements}

We used biomedical data acquisition software (Poly, Inspektor Research Systems BV, Amsterdam, The Netherlands) to store Qufp, Qpre and Qpost with a $200 \mathrm{~Hz}$ sampling rate. The exact span of the TPUD measurements was marked in the registration. Qp/Qs ratio was calculated using the following equation: Qp/Qs $=($ Qufp+Qpre-Qpost $) /$ Qufp. Flow probes were calibrated before each experiment. Before every measurement, the adequacy of signal strength of the probes was checked. Additional acoustic gel was applied in case of decreased signal strength (quality $<0.7$ on analog meter in “TEST" mode).

\section{Data analysis}

Shunt detection was assessed using sensitivity and specificity. The positive likelihood ratio was calculated as sensitivity/(1-specificity) and the negative likelihood ratio as (1-sensitivity)/specificity. The Pearson correlation was used to compare Qp/Qs measured by TPUD $[\mathrm{Qp} / \mathrm{Qs}($ tpud)] and Qp/Qs measured by flow probes [Qp/Qs(ufp)]. In addition, the data were analyzed using the method described by Bland-Altman for repeated measurements [27]. Mean bias was defined 
A

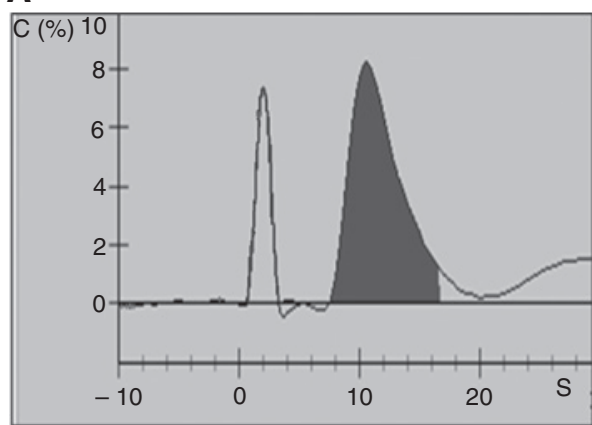

B

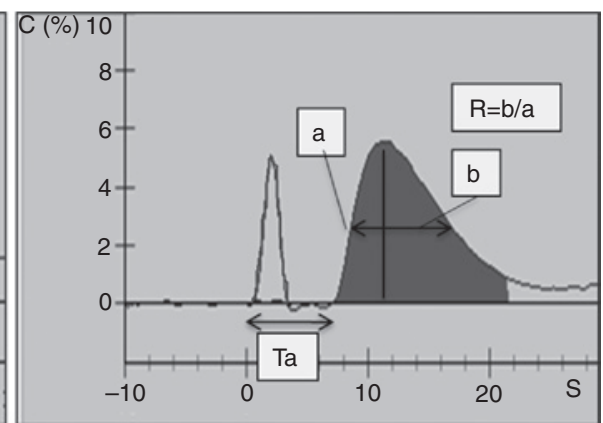

Figure 3: Examples of TPUD curves (dark gray) without (A) and with (B) a left-to-right shunt.

The initial (light gray) curves represent the change in ultrasound velocity recorded by the venous sensor. Ta=Appearance time [time from the beginning of the venous injection to the beginning of the dilution curve(s)], $a=$ the upslope of the curve at half maximum height (\%) and $\mathrm{b}=$ full width at half maximum height(s).

as the mean difference between $\mathrm{Qp} / \mathrm{Qs}(\mathrm{tpud})$ and $\mathrm{Qp} / \mathrm{Qs}(\mathrm{ufp})$. The mean bias was plotted against the mean $Q p / Q s\{[Q p / Q s(u f p)+Q p /$ Qs(tpud)]/2\}. Precision was represented by limits of agreement (LOA). A mixed effects linear model was used to estimate the variance component due to lamb $\left(\sigma_{\mathrm{L}}^{2}\right)$ and the residual variance $\left(\sigma_{\mathrm{R}}^{2}\right)$. The LOA were then calculated as mean (bias) $\pm 1.96 \times V\left(\sigma_{L}^{2}+\sigma_{R}^{2}\right)$. Percentage error was calculated as $100 \times[1.96 \times \mathrm{SD}$ (bias)/mean Qp/Qs(ufp)]. Qp/Qs changes of both methods after shunt opening/closure and after hemorrhage were calculated. Correlation between changes in Qp/Qs(tpud) and Qp/Qs(ufp) was determined. SPSS 20.0 for Windows ${ }^{\circledR}$ (SPSS Inc., Chicago, IL, USA) was used for statistical analysis.

\section{Results}

A total of 72 measurement sessions were performed and analyzed. Qufp ranged from 0.61 to $1.75 \mathrm{~L} / \mathrm{min}$ (mean $1.10 \mathrm{~L} / \mathrm{min}$ ) and from 0.47 to $1.46 \mathrm{~L} / \mathrm{min}$ (mean $0.91 \mathrm{~L} /$ min) with the shunt closed and open, respectively. With the shunt closed mean Qp/Qs(ufp) was $1.0 \mathrm{~L} / \mathrm{min}(0.8-$ $1.1 \mathrm{~L} / \mathrm{min}$ ); with the shunt open mean Qp/Qs(ufp) was $1.8 \mathrm{~L} / \mathrm{min}(1.3-2.6 \mathrm{~L} / \mathrm{min})$. Table 1 shows the results for shunt detection by TPUD: positive predictive value was $86 \%$, negative predictive value $100 \%$, sensitivity $100 \%$

Table 1: Shunt detection using transpulmonary ultrasound dilution (TPUD).

\begin{tabular}{lrrrlr}
\hline & Shunt & No shunt & Total & & PPV \\
\cline { 1 - 2 } Detection & 37 & 6 & 43 & NPV & $86 \%$ \\
No detection & 0 & 29 & 29 & Sensitivity & $100 \%$ \\
Total & 37 & 35 & 72 & Specificity & $83 \%$ \\
& & & & LR+ & 5.8 \\
& & & & LR- & 0.0 \\
\hline
\end{tabular}

$\mathrm{PPV}=$ positive predictive value, $\mathrm{NPV}=$ negative predictive value, $L R+=$ positive likelihood ratio, $L R-=$ negative likelihood ratio. and specificity $83 \%$. The positive likelihood ratio was 5.8 , and the negative likelihood ratio was 0.0. Figure 4 shows an overview of the mean cardiac output measured by the transit-time flow probes (Qufp) and the TPUD (COtpud) (A), and the mean Qp/Qs measured by the transit-time flow probes [Qp/Qs(ufp)] and the TPUD [Qp/ Qs(tpud)] (B) during the different phases of the experiment. Comparisons between all detected absolute values of Qp/Qs(tpud) and Qp/Qs(ufp) (including six false positive measurements) and changes in Qp/Qs after opening/ closure of the shunt and hemorrhage between the two methods are shown in Figure $5 \mathrm{~A}$ and $\mathrm{B}$, respectively. The Pearson correlation for absolute values between the two methods was $\mathrm{r}^{2}=0.40(\mathrm{P}=0.01)$; the correlation for changes was $\mathrm{r}^{2}=0.82(\mathrm{P}=0.01)$. The Bland-Altman analysis for absolute values between $Q p / Q p(t p u d)$ and $Q p /$ Qs(ufp) showed an overall mean bias (SD) of 0.1 with LOA \pm 0.6 and a percentage error of $34.8 \%$ (Figure 6 ). There was no significant correlation between the bias in $\mathrm{Qp} /$ Qs measured by both methods and the cardiac output $\left(r^{2}=0.13\right)$. Bias and shunt size correlated negatively $\left(r^{2}=0.50\right)$.

\section{Discussion}

Our study showed that the TPUD method is capable of detecting left-to-right shunts with a high sensitivity and specificity. However, quantification of the shunt (expressed as Qp/Qs) correlates moderately with Qp/Qs measured by flow probes.

The use of algorithms and changes in dilution curves in the presence of cardiac shunts for the detection and quantification of shunts date from the 1950s [26, 28, 29]. However, TPUD is the first hemodynamic monitoring 

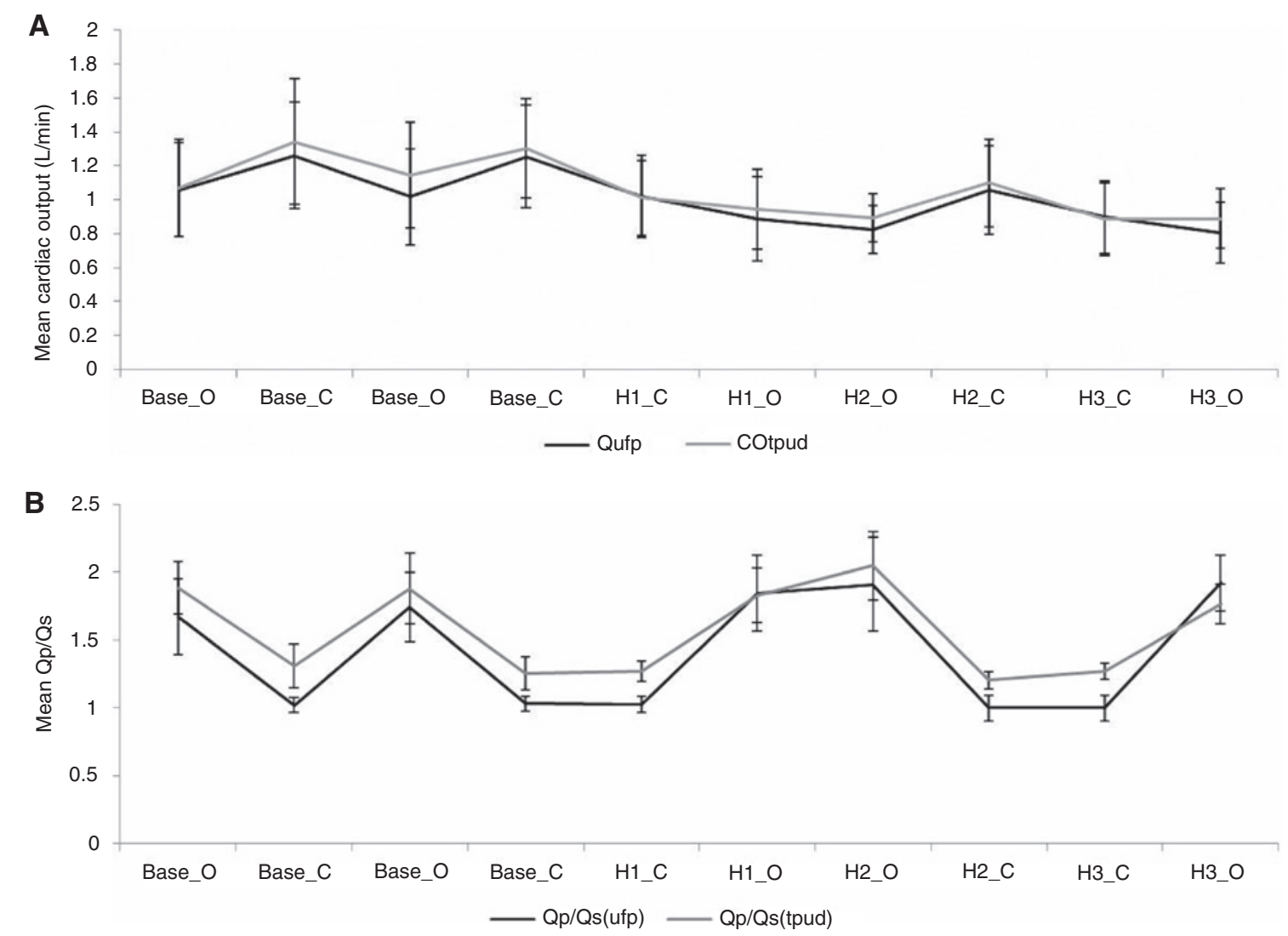

Figure 4: Overview of the mean cardiac output measured by the transit-time flow probes (Qufp) and the TPUD (COtpud) (A), and the mean Qp/Qs measured by the transit-time flow probes (Qp/Qs(ufp) and the TPUD [Qp/Qs(tpud)] (B) during the different phases of the experiment. Error bars represent the standard deviation. $\mathrm{O}=$ open shunt, $\mathrm{C}=$ closed shunt, $\mathrm{H}=$ hemorraghe.

device with the ability to detect and quantify shunts usable in a neonatal population as it uses no toxic indicator and does not require specific large catheters. In neonatal intensive care, patients present with a range of (congenital) cardiac anomalies, with the highest incidence of PDA, especially in preterm infants.

Early diagnosis of hsPDA is important for consideration of initiation of therapy and prevention of PDA-associated morbidity. As mentioned previously, the definition of hsPDA is not (yet) standardized. Echocardiographic parameters used for defining hsPDA are left atrial-to-aortic root (LA/Ao) ratio and ductal diameter, sometimes in combination with pulsed and color Doppler parameters (diastolic disturbances in main pulmonary artery, shunt direction, etc) and reversed flow in the descending aorta. Only a few studies mention the $\mathrm{Qp} / \mathrm{Qs}$ ratio as a parameter [7, 30]. Evans classified Qp/Qs ratio $<1.5$ as small, Qp/ Qs ratio 1.5-2.0 as moderate and Qp/Qs ratio $>2$ as large shunt in preterm infants $<30$ weeks [7].

The sensitivity of shunt detection using TPUD was $100 \%$ with a specificity of $83 \%$ as a result of six false positive measurements, findings that are in agreement with current reports in the literature: Shih found a success rate in detecting intracardiac right-to-left shunts of $86 \%$ when Qp/Qs $<0.8$ and $100 \%$ when Qp/Qs $<0.5$ in a piglet model using TPUD compared to UFP [31]. Boehne reported a sensitivity of $100 \%$ and specificity of $92.8 \%$ in 20 patients with a left-to-right shunt (threshold Qp/Qs>1.3) when comparing TPUD and cardiac catheterization [32]. Lindberg confirmed these results in 21 children with preoperatively intracardiac left-to-right shunts: sensitivity $100 \%$ and specificity $92 \%$ [24]. Although the specificity in our study is comparable with the mentioned studies, our false positive rate might even be less: despite the fact that the shunt was visibly closed in one-measurement session, there was still a Qp/Qs(ufp) of 1.15 which was detected by TPUD (Figure 5A). In addition, it is plausible that some lambs presented with intracardiac left-to-right shunts (i.e. patent foramen ovale, septal defects), which we did not rule out by echocardiography. These shunts could be detected by TPUD but missed with the current calculation of Qp/Qs(ufp). 

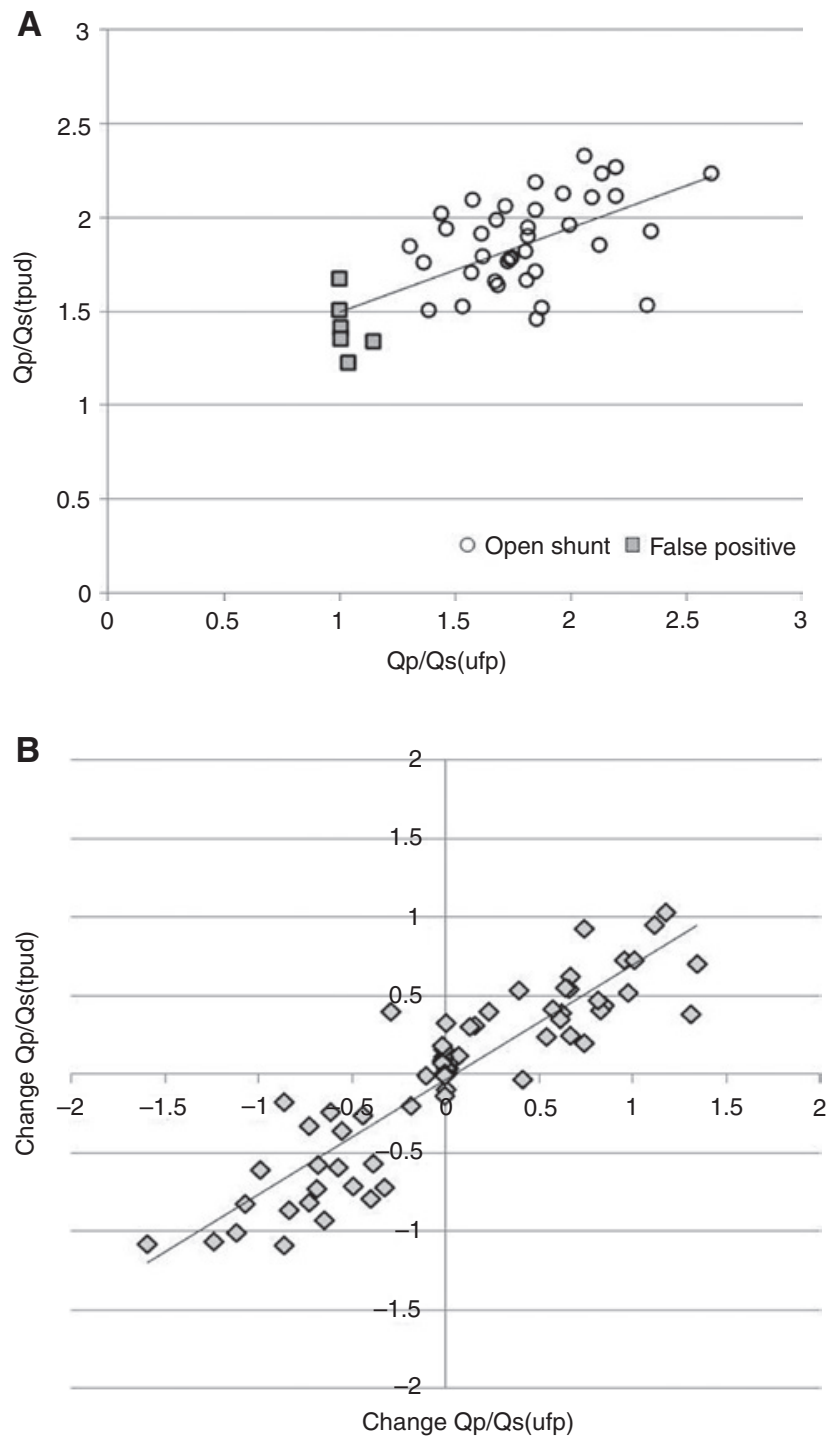

Figure 5: The Pearson correlation between $Q p / Q s(t p u d)$ and $Q p /$ Qs(ufp) for all detected measurements ( $A$ ) and for changes in $Q p /$ Qs(tpud) and Qp/Qs(ufp) after shunt opening/closure and after hemorrhage (B).

Gray squares=false positives; white circles=Qp/Qs(ufp) measurements with open shunt.

In clinical practice, the incidence of PDA in (extremely) preterm infants varies between $20 \%$ and $60 \%$. As the positive likelihood ratio in our study is 5.8, the post-test probability of having a left-to-right shunt with a positive test will increase from $59 \%$ to $89.5 \%$. This suggests that TPUD is an accurate screening method for diagnosing a left-toright shunt. A small number of patients without PDA will also test positive and might be unnecessary exposed to an additional echocardiography at that time-point. However, the diagnosis of PDA by TPUD should not only solely be based on an increased Qp/Qs ratio, but also on changes in cardiac output (systemic blood flow) and hemodynamic volumes like active circulating volume, central blood volume and total end-diastolic blood volume [33]. In the presence of a left-to-right shunt, systemic blood flow will decrease (as a result of ductal steal) and there will be an increase in central blood volume [33] and especially in total end-diastolic volume (as a result of an increased left atrium volume).

Besides detection, TPUD also quantifies the shunt fraction by calculating the Qp/Qs ratio. We opted to use all the detected Qp/Qs ratios (inclusively the false positive ones) for further analysis as it is not possible - based on the obtained values of the TPUD - to clinically distinct between true and false positive measurements. Correlation between the detected Qp/Qs(tpud) and Qp/Qs(ufp) was moderate. In this study, we found an overall mean bias of 0.1 with LOA of \pm 0.6 when comparing Qp/Qs(tpud) and Qp/Qs(ufp), resulting in an overall good accuracy and moderate precision (percentage error 34.8\%). There is a noticeable trend that TPUD overestimates Qp/Qs in small shunts and underestimates Qp/Qs in large shunts. These finding are possibly due to an inaccuracy in the current algorithm which is used to predict the area under the normal dilution curve. This would also explain the deviation in bias in relation to the shunt size. Also, the lack of information on possible intracardiac shunts (i.e. patent foramen ovale) could influence shunt flow in a dynamic manner and, therefore, the relationship between the two Qp/Qs measurements. Finally, the moderate precision could be influenced by the absence of actual shunt flow measurement, as it is not possible for flow probes to measure flow through a vascular Goretex graft. Flow probes were placed before and after the shunt insertion on the descending aorta. Subsequently, Qp/Qs(ufp) was calculated using three different flow probe measurements which might increase the chance of error. This is substantiated by the fact that after shunt closure, Qp/Qs(ufp) ratios varied between 0.8 and 1.1, while they should ideally approximate 1 .

Lindberg also determined Qp/Qs by UFP in eight of his 21 patients and found a mean bias of -0.4 with a percentage error of $54 \%$ when comparing $\mathrm{Qp} / \mathrm{Qs}$ (tpud) and $\mathrm{Qp} / \mathrm{Qs}(\mathrm{ufp})$ [24]. In contrast, in a group of post-cardiac surgery children with very small residual shunts $(\mathrm{Qp} / \mathrm{Qs}$ $0.8-1.3$ ), quantification of shunts by TPUD was in agreement with echocardiographic estimates [25]. Based on our results, we believe that single absolute Qp/Qs(tpud) values should not be used to quantify left-to-right shunts in the clinical setting. An adaptation of the algorithm for calculation Qp/Qs by the manufacturer might possibly enhance the precision of the method. However, TPUD is a 


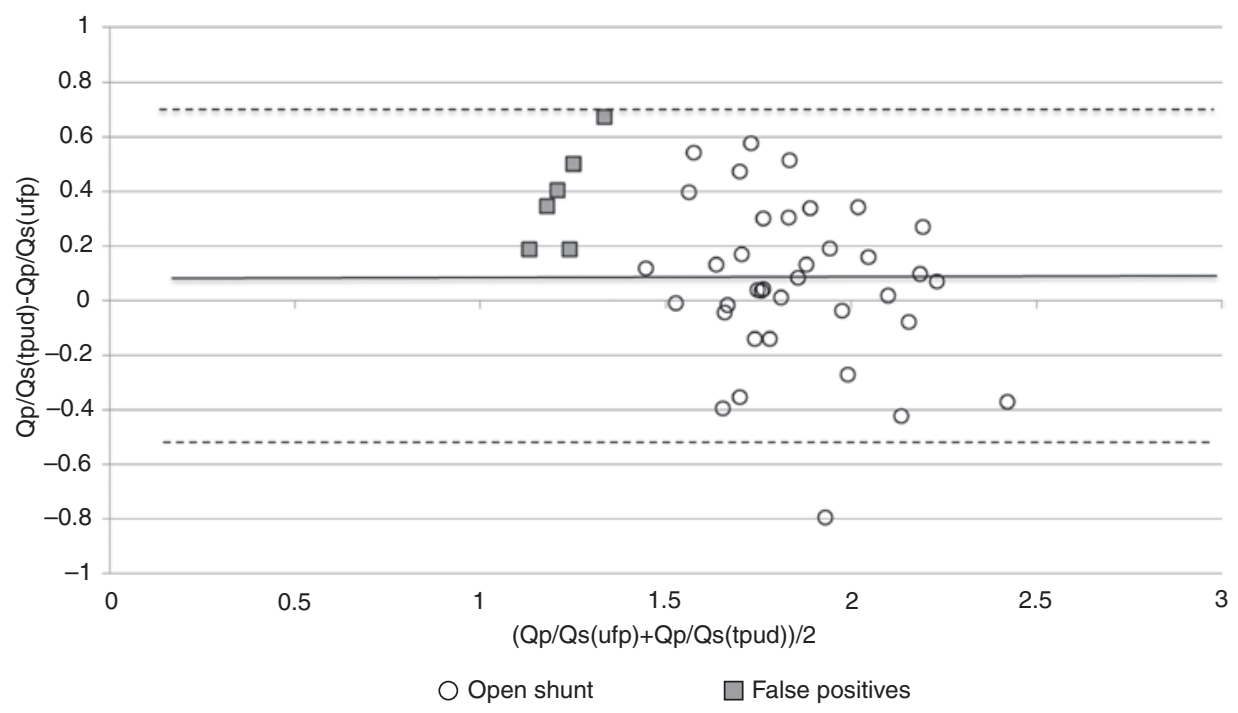

Figure 6: The Bland-Altman plot for the assessment of agreement between Qp/Qs measured by transpulmonary ultrasound dilution [Qp/ Qs(tpud)] and Qp/Qs measured by flow probes [Qp/Qs(ufp)].

The solid line represents the mean bias and the dotted lines represent the limits of agreement for all data [false positives (gray squares) and open shunt (white circles)].

reliable device to detect (even small) changes in Qp/Qs as shown in Figure 5B.

Despite this current lack of precision for absolute Qp/Qs estimates, we believe that TPUD has potential for future neonatal clinical use. The major advantage is the possibility of simultaneous monitoring of various hemodynamic parameters at bedside. Repetitive measurements could reveal changes in cardiac output, volumes and Qp/ Qs ratios and optimize the moment for further diagnostic evaluation. When used in clinical practice, it is obvious that a left-to-right shunt detected by TPUD might be caused by a PDA, and also by other intra- (open foramen ovale, ventricle septum defect) or extracardiac (aortopulmonary shunt) shunts. Therefore, echocardiography is indispensible to exclude congenital heart diseases before starting a treatment for ductal closure. After initiating medical therapy for ductal closure, repetitive measurements can provide information on the efficacy of therapy as TPUD might be used as a monitoring device. Further research in this clinical setting is warranted.

\section{Limitations}

As aforementioned, we did not evaluate the presence of possible intracardiac shunts by echocardiography. Due to the insertion of vascular grafts of 4-6 $\mathrm{mm}$, we were not able to perform measurements with very small shunts (Qp/Qs 1.1-1.3), and data on detection and quantification of Qp/Qs by TPUD in small shunts are, therefore, lacking.

\section{Conclusions}

We conclude that TPUD is a hemodynamic monitoring device that is able to detect (left-to-right) shunts with highpredictive value. Quantification of the shunt (expressed as Qp/Qs ratio) by TPUD is possible but lacks precision in its current application; however, trending ability to detect changes in Qp/Qs is high. We believe that the TPUD hemodynamic monitoring system can be used in the neonatal period as an initial screening tool to detect (left-to-right) shunts and monitor ductal closure after starting the initial medical treatment.

Acknowledgments: We would like to thank J.J.M. Menssen, Laboratory of Clinical Physics of the Department of Pediatrics, Radboud University Nijmegen Medical Centre; A.E.J. Hanssen, Animal Laboratory of the Radboud University Nijmegen and A.F.J. de Haan, MSc, Department of Epidemiology, Statistics and Health Technology Assessment of the Radboud University Nijmegen for their outstanding support. Our research group received financial support for the technical realization of this experiment from Transonic Systems Inc., Ithaca, NY, USA and Pulsion Medical Systems, Munich, Germany.

\section{References}

[1] Project lotV-OTND. The vermont-oxford trials network: very low birth weight outcomes for 1990. Pediatrics. 1993;91:540-5. 
[2] Reller MD, Rice MJ, McDonald RW. Review of studies evaluating ductal patency in the premature infant. J Pediatr. 1993;122:S59-62.

[3] Ichihashi K, Shiraishi H, Endou H, Kuramatsu T, Yano S, Yanagisawa M. Cerebral and abdominal arterial hemodynamics in preterm infants with patent ductus arteriosus. Acta Paediatr Jpn. 1990;32:349-56.

[4] Kluckow M, Evans N. Ductal shunting, high pulmonary blood flow, and pulmonary hemorrhage. J Pediatr. 2000;137:68-72.

[5] Hamrick SE, Hansmann G. Patent ductus arteriosus of the preterm infant. Pediatrics. 2010;125:1020-30.

[6] Evans N, Kluckow M. Early ductal shunting and intraventricular haemorrhage in ventilated preterm infants. Arch Dis Child Fetal Neonatal Ed. 1996;75:F183-6.

[7] Evans N. Diagnosis of the preterm patent ductus arteriosus: clinical signs, biomarkers, or ultrasound? Semin Perinatol. 2012;36:114-22.

[8] Cambonie G, Dupuy AM, Combes C, Vincenti M, Mesnage R, Cristol JP. Can a clinical decision rule help ductus arteriosus management in preterm neonates? Acta Paediatr. 2012;101:e213-8.

[9] Kwinta P, Rudzinski A, Kruczek P, Kordon Z, Pietrzyk JJ. Can early echocardiographic findings predict patent ductus arteriosus? Neonatology. 2009;95:141-8.

[10] Sehgal A, McNamara PJ. Does echocardiography facilitate determination of hemodynamic significance attributable to the ductus arteriosus? Eur J Pediatr. 2009;168:907-14.

[11] Zonnenberg I, de Waal K. The definition of a haemodynamic significant duct in randomized controlled trials: a systematic literature review. Acta Paediatr. 2012;101:247-51.

[12] Jain A, Shah PS. Diagnosis, evaluation, and management of patent ductus arteriosus in preterm neonates. JAMA Pediatr. 2015;169:863-72.

[13] Kluckow M, Seri I, Evans N. Functional echocardiography: an emerging clinical tool for the neonatologist. J Pediatr. 2007;150:125-30.

[14] Kluckow M, Evans N. Low superior vena cava flow and intraventricular haemorrhage in preterm infants. Arch Dis Child Fetal Neonatal Ed. 2000;82:F188-94.

[15] Krivitski NM, Kislukhin VV, Thuramalla NV. Theory and in vitro validation of a new extracorporeal arteriovenous loop approach for hemodynamic assessment in pediatric and neonatal intensive care unit patients. Pediatr Crit Care Med. 2008;9:423-8.

[16] Eremenko AA, Safarov PN. Flow-regulated extracorporeal arteriovenous tubing loop for cardiac output measurements by ultrasound velocity dilution: validation in post-cardiac surgery intensive care unit patients. ASAIO J. 2010;56:522-6.

[17] Tsutsui M, Matsuoka N, Ikeda T, Sanjo Y, Kazama T. Comparison of a new cardiac output ultrasound dilution method with thermodilution technique in adult patients under general anesthesia. J Cardiothorac Vasc Anesth. 2009;23:835-40.

[18] Gleed RD, Callahan M, Smith T. Validation of novel ultrasound dilution cardiac output method for pediatric and neonatal patients. Eur Soc Intensive Care Med. 2006;32:S172.

[19] Crittendon I, 3rd, Dreyer WJ, Decker JA, Kim JJ: Ultrasound dilution: an accurate means of determining cardiac output in children. Pediatr Crit Care Med. 2012;13:42-6.
[20] Boehne M, Baustert M, Paetzel V, Koditz H, Schoof S, Happel CM, et al. Determination of cardiac output by ultrasound dilution technique in infants and children: a validation study against direct fick principle. Br J Anaesth. 2014;112:469-76.

[21] de Boode WP, van Heijst AF, Hopman JC, Tanke RB, van der Hoeven HG, Liem KD. Cardiac measurement using an ultrasound dilution method: a validation study in ventilated piglets. Pediatr Crit Care Med. 2010;11:103-8.

[22] Vrancken SL, de Boode WP, Hopman JC, Singh SK, Liem KD, van Heijst AF. Cardiac output measurement with transpulmonary ultrasound dilution is feasible in the presence of a left-to-right shunt: a validation study in lambs. $\mathrm{Br}$ J Anaesth. 2012;108:409-16.

[23] Vrancken SL, de Boode WP, Hopman JC, Looijen-Salamon MG, Liem KD, van Heijst AF. Influence of lung injury on cardiac output measurement using transpulmonary ultrasound dilution: a validation study in neonatal lambs. $\mathrm{Br}$ J Anaesth. 2012;109:870-8.

[24] Lindberg L, Johansson S, Perez-de-Sa V. Validation of an ultrasound dilution technology for cardiac output measurement and shunt detection in infants and children. Pediatr Crit Care Med. 2014;15:139-47.

[25] Saxena R, Krivitski N, Peacock K, Durward A, Simpson JM, Tibby SM. Accuracy of the transpulmonary ultrasound dilution method for detection of small anatomic shunts. J Clin Monit Comput. 2015;29:407-14.

[26] Mook GA, Zijlstra WG. Quantitative evaluation of intracardiac shunts from arterial dye dilution curves. Demonstration of very small shunts. Acta Med Scand. 1961;170:703-15.

[27] Bland JM, Altman DG. Agreement between methods of measurement with multiple observations per individual. J Biopharm Stat. 2007;17:571-82.

[28] Carter SA, Bajec DF, Yannicelli E, Wood EH. Estimation of leftto-right shunt from arterial dilution curves. J Lab Clin Med. 1960;55:77-88.

[29] Morrow AG, Oldham HN, Callard GM, Braunwal E. Assessment of operative results in congenital heart disease by intraoperative indicator-dilution curves. Circulation. 1966;33:263-9.

[30] El Hajjar M, Vaksmann G, Rakza T, Kongolo G, Storme L. Severity of the ductal shunt: a comparison of different markers. Arch Dis Child Fetal Neonatal Ed. 2005;90:F419-22.

[31] Shih A, Maisenbacher III H, Vigani A, Estrada A, Pogue B, Berry $C$, et al. Ultrasound dilution technique as a minimally invasive way to detect intracardiac shunt. Crit Care Med. 2011;39:61. [abstract 0683].

[32] Boehne M, Baustert M, Happel CM, Schoff S, Hohmann D, Paetzel V, et al. Novel ultrasound dilution technique detects left-to-right shunts with high accuracy in children. Pediatr Res. 2010;68:117. [abstract 224].

[33] Boehne M, Schmidt F, Witt L, Koditz H, Sasse M, Sumpelmann R, et al. Comparison of transpulmonary thermodilution and ultrasound dilution technique: movel insights into volumetric parameters from an animal model. Pediatr Cardiol. 2012;33:625-32.

The authors stated that there are no conflicts of interest regarding the publication of this article. 